\title{
Clinical Characteristics and Treatment Outcomes of Patients with Eosinophilic Esophagitis and Eosinophilic Gastroenteritis
}

\author{
Eiko Okimoto Norihisa Ishimura Shunji Ishihara \\ Second Department of Internal Medicine, Shimane University Faculty of Medicine, Izumo, Japan
}

\begin{abstract}
Keywords
Eosinophilic esophagitis - Eosinophilic gastroenteritis . Eosinophilic gastrointestinal disorders · Proton pump inhibitor · Food-elimination diet
\end{abstract}

\begin{abstract}
Background: Eosinophilic esophagitis (EoE) and eosinophilic gastroenteritis (EGE), part of the spectrum of eosinophilic gastrointestinal disorders (EGID), share pathogenic similarities. We examined differences regarding clinical characteristics and treatment outcomes between EoE and EGE cases. Methods: Two-hundred fifteen EGID patients, including 181 with EoE and 34 with EGE, diagnosed at Shimane University Hospital between February 2011 and March 2019 were enrolled. Information regarding clinical parameters and treatment outcomes was reviewed. Results: EoE showed significant male predominance $(82.3 \%)$ as compared with EGE (50.0\%) $(p<0.001)$. Furthermore, patients with EoE were significantly older and had a higher body mass index (24.8 \pm 4.0 vs. $22.2 \pm 4.3, p<0.05$ ). Over $90 \%$ of the EoE patients were initially given proton pump inhibitor (PPI) treatment, of whom 73.2\% showed clinical and histological remission. Vonoprazan, a more potent acid inhibitor than PPI, was effective in two-thirds of the nonresponsive EoE patients initially treated with a PPI. In contrast, oral glucocorticoid administration was mainly given to patients with EGE (58.8\%). Of 13
\end{abstract}

EGE patients treated with a food-elimination diet, responsible foods were successfully identified in 9, with 7 controlled in a state of remission without glucocorticoid therapy. Conclusions: We found different clinical characteristics and treatment strategies in the present EoE and EGE cases. Most of the EoE patients responded to and were maintained by acid suppressive therapy, using PPI or vonoprazan. For EGE patients, glucocorticoid administration was mainly used though food-elimination diet therapy also showed beneficial effects.

(C) 2020 The Author(s)

Published by S. Karger AG, Basel

\section{Introduction}

Eosinophilic gastrointestinal disorders (EGID) are chronic immune-mediated conditions characterized by dense eosinophilic infiltration of the gastrointestinal (GI) tract without any known cause of eosinophilia, such as parasitic infection or inflammatory bowel disease $[1,2]$. Depending on the involved GI tract, EGID are divided into eosinophilic esophagitis (EoE) and eosinophilic gastroenteritis (EGE), with EoE involving only the esophagus, while EGE involves any part of the GI tract. Although the pathogenesis remains to be fully elucidated, $T$ helper 2 (Th2) immune response related to food and/or aeroallergens may play a central role in dysfunctional eosino- karger@karger.com www.karger.com/dig

Karger $\stackrel{\text { ' }}{5}$

GOPEN ACCESS
(C) 2020 The Author(s)

Published by S. Karger AG, Basel

This is an Open Access article licensed under the Creative Commons Attribution-NonCommercial-4.0 International License (CC BY-NC) (http://www.karger.com/Services/OpenAccessLicense), applicable to the online version of the article only. Usage and distribution for commercial purposes requires written permission.
Norihisa Ishimura

Second Department of Internal Medicine, Shimane University Faculty of Medicine 89-1, Enya-cho

Izumo 693-8501 (Japan)

ishimura@med.shimane-u.ac.jp 
philic inflammation in the GI tract [3-5]. However, differences regarding pathophysiology factors between EoE and EGE have not been clarified.

Over the past 2 decades, both incidence and prevalence of EoE have been rapidly increasing, especially in Western countries, though EGE remains a rare condition $[6,7]$. In Japan, while EGE has been shown to be more prevalent and EoE noted as a very rare condition in various studies [8-10], the latter has begun to show a gradually increasing incidence in recent years [11-13]. Several studies have demonstrated that clinical features of EoE patients in Japan are similar to those in Western countries though the clinical presentation of EoE is milder in Japanese cases $[14,15]$, as severe complications, such as esophageal stricture and perforation, are rarely observed [16]. However, the long-term course of EoE after initial treatment in Japanese patients remains to be elucidated. Furthermore, there are a very little data concerning the clinical features of EGE focusing on the differences between Japanese and Western populations [10]. Although differences in GI symptoms were seen between Caucasians and Asians in EGE, genetic and environmental studies remain to be fully elucidated. In the present study, we aimed to clarify differences in clinical features, including long-term treatment outcomes, between EoE and EGE patients treated in Japan.

\section{Methods}

\section{Patients and Data Collection}

We retrospectively enrolled 215 patients with EGID, including 181 with EoE and 34 with EGE, who were diagnosed and/or treated at Shimane University Hospital between February 2011 and March 2019. EoE was defined clinically by symptoms of esophageal dysfunction, such as dysphagia, and histologically by the presence of esophageal eosinophilia, which is classified in the current guidelines as $>15$ eosinophils per high-power field in at least 1 esophageal biopsy specimen [17]. EGE was defined by the presence of GI symptoms associated with pathological eosinophilic infiltration of $>20$ eosinophils per high-power field in the GI tract and/or eosinophilic ascites fluid $[18,19]$. Cases with asymptomatic esophageal eosinophilia or secondary causes of EGID were excluded.

Information regarding clinical parameters, including age, gender, BMI, concurrent allergic disease, presenting symptoms, laboratory testing, endoscopic findings, and involved GI tract, in the enrolled patients was reviewed and then compared between the EoE and EGE patients. In addition, treatments and outcomes for each patient were also reviewed.

\section{Treatment Algorithm for Patients with EoE and EGE}

The treatment algorithm based on acid-suppression therapy used for patients with EoE at our institution is shown in Figure 1. When EoE is diagnosed after exclusion of other causes, a standard dose of a proton pump inhibitor (PPI) is given as the first-line treatment, and then an endoscopic biopsy is conducted 8 weeks later to evaluate response. If the patient does not respond well to PPI therapy, we usually change to treatment with vonoprazan, a potassium-competitive acid blocker launched in 2015 and stronger acid suppressant than PPIs or topical steroids [12]. For patients who respond to the administered PPI, that treatment is continued. One year later, an endoscopic biopsy is again performed to reevaluate esophageal eosinophilia. During the course of treatment, when a patient shows a relapsing symptom and/or esophageal eosinophilia, vonoprazan or topical steroid treatment is given, depending on the condition of the individual patient. When no relapse has occurred after 1 year of PPI therapy, its discontinuance is considered and annual follow-up endoscopic examinations are conducted.

In contrast to EoE, no standard treatment strategy for EGE has been established and no guidelines presented because the prevalence is extremely rare as compared with EoE, especially in Western countries. The most widely used treatment modality is systemic glucocorticoid administration [9].

\section{Statistical Analysis}

Data are presented as number of cases and/or percentages for categorical data. Statistical analysis of categorical data was performed using $\chi^{2}$ test or Fisher's exact test, while a Mann-Whitney $\mathrm{U}$ test was used for numerical values to compare the clinical characteristics between the EoE and EGE patients. A $p$ value of $<0.05$ was considered to be statistically significant.

\section{Results}

\section{Clinical Characteristics of EoE and EGE Patients}

Clinical characteristics of patients diagnosed with EoE or EGE are shown in Table 1. The mean age in years for both groups was in the 40s though patients with EoE were significantly older $(47.5 \pm 12.0$ vs. $41.6 \pm 21.0$ years, $p<$ $0.05)$. In addition, the EoE group showed significant male predominance $(82.3 \%)$ in comparison with the EGE group $(50.0 \%)(p<0.001)$. Also, the EoE patients had a greater BMI ( $24.8 \pm 4.0$ vs. $22.2 \pm 4.3, p<0.05)$, while obesity, defined as BMI $\geq 25 \mathrm{~kg} / \mathrm{m}^{2}$, was also more prevalent in patients with EoE than in those with EGE. Although $>70 \%$ of patients in both groups had concurrent allergic diseases, food allergy was more frequently noted in the EGE group (38.2 vs. 9.4\%, $p<0.001$ ). Interestingly, allergy to seafoods including shellfish was most common, followed by fruits in both groups, suggesting that the frequency of food allergy was more frequently observed in EGE groups, while type of the food antigens was comparable between the groups.

The most frequently reported symptom in patients with EoE was dysphagia (66.9\%), followed by heartburn (43.1\%), while few had a history of food impaction. In 


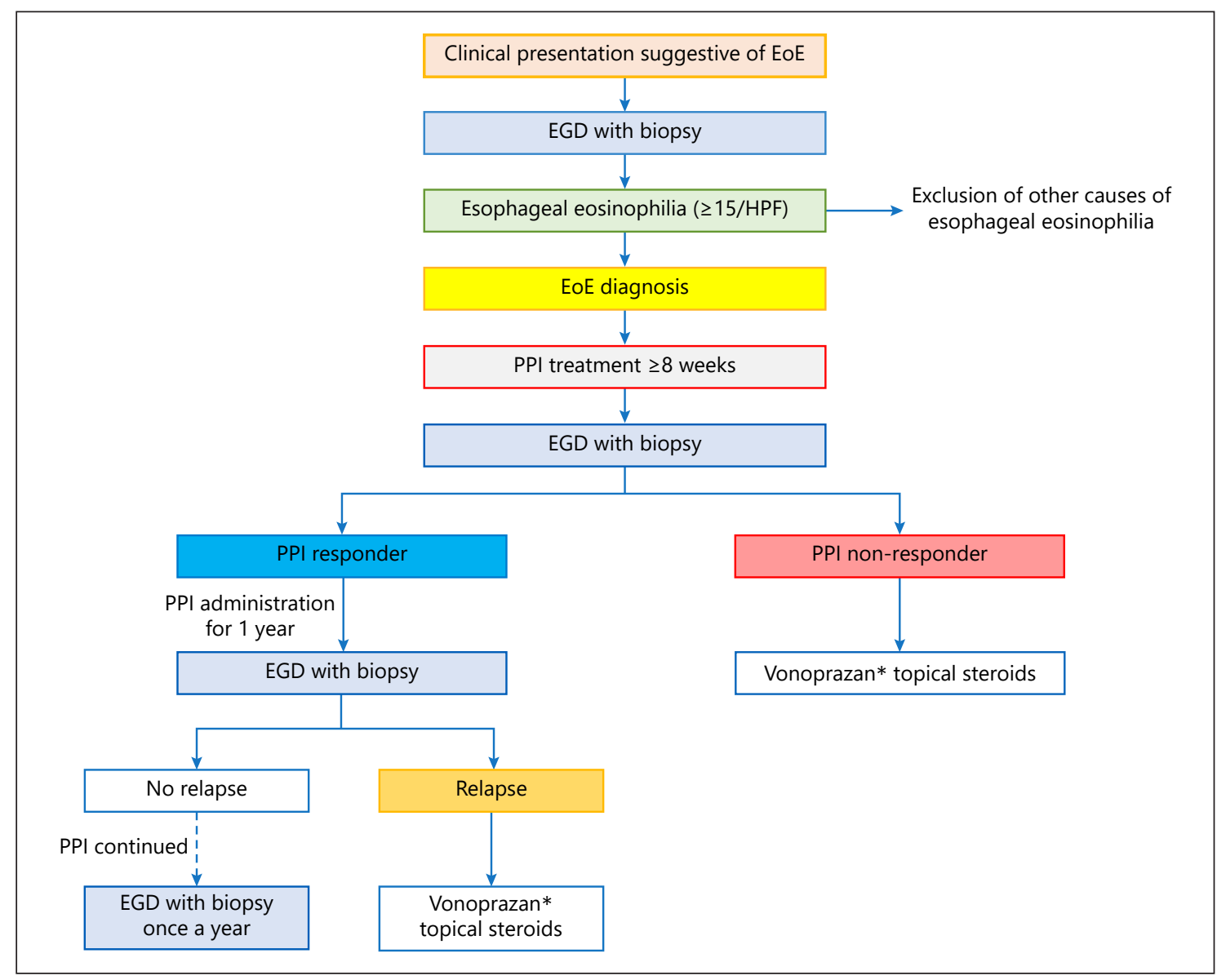

Fig. 1. Diagnosis and treatment algorithm based on acid-suppression therapy for patients with EoE. Vonoprazan, a novel potassium-competitive acid blocker, was available from 2015 and used as a treatment option for EoE. EoE, eosinophilic esophagitis; EGD, esophagogastroduodenoscopy; HPF, high-power field; PPI, proton pump inhibitor.

contrast, the most frequently reported symptom in patients with EGE was abdominal pain (61.8\%), followed by diarrhea (47.1\%). Laboratory test results showed no significant differences between EoE and EGE in regard to peripheral eosinophilia, CRP, and total IgE (Table 2). The rate of positive for Helicobacter pylori was lower in patients with EGE than EoE though the difference was not significant. As for endoscopic findings, linear furrows, rings, and whitish exudate were frequently observed in patients with EoE, and at least one of those findings was seen in nearly all of the cases. In contrast, no characteristic endoscopic findings, including erosion, edema, and redness, were observed in the EGE patients. Endoscopic findings in patients with esophageal involvement of EGE $(n=9)$ were comparable with those with EoE.

Eosinophilic Esophagitis and Gastroenteritis
Eosinophilic infiltration was limited to the esophagus in the EoE group, while that was observed in one or more parts of the GI tract in patients with EGE. The most frequently noted area with GI lesion involvement was the small intestine (76.5\%), followed by the colon (55.9\%) and stomach $(41.2 \%)$, while $26.5 \%$ also had esophageal lesions. Ascites was observed in $17.4 \%$ of patients with EGE, whereas that was not observed in any of the EoE patients.

\section{Treatments for and Outcomes of Patients with EoE}

To date, PPI administration is considered to be the primary pharmacologic choice for EoE in Japan. In the present cohort, over $90 \%$ of the present patients with EoE were initially treated with a PPI, of whom $73.2 \%$ showed clinical and histological remission (Fig. 2). Vonoprazan 
Table 1. Clinical features of EoE and EGE

\begin{tabular}{llcc}
\hline & EoE $(n=181)$ & EGE $(n=34)$ & $p$ value \\
\hline Age, years, mean \pm SD & $47.5 \pm 12.0$ & $41.6 \pm 21.0$ & $<0.05$ \\
Male sex, \% & 82.3 & 50.0 & $<0.001$ \\
BMI, kg/m ${ }^{2}$, mean \pm SD & $24.8 \pm 4.0$ & $22.2 \pm 4.3$ & $<0.05$ \\
Obesity $\left(\mathrm{BMI} \geq 25 \mathrm{~kg} / \mathrm{m}^{2}\right), \%$ & 44.0 & 21.4 & $<0.05$ \\
Concurrent allergic disease, \% (duplicates counted) & 75.7 & 70.6 & 0.53 \\
$\quad$ Allergic rhinitis & 45.9 & 38.2 & 0.41 \\
Bronchial asthma & 26.0 & 17.6 & 0.30 \\
Atopic dermatitis & 13.8 & 20.6 & 0.31 \\
Food allergy* & 9.4 & 38.2 & $<0.001$ \\
Symptoms, \% (duplicates counted) & & & $<0.001$ \\
Dysphagia & 66.9 & 14.7 & $<0.001$ \\
Heartburn & 43.1 & 61.8 & $<0.001$ \\
Abdominal pain & 6.1 & 47.1 & $<0.001$ \\
Diarrhea & 1.1 & 29.4 & 0.52 \\
Peripheral eosinophilia, \% & 18.5 & 14.7 & 0.67 \\
CRP elevation, \% & 12.1 & 71.0 & 0.52 \\
Total IgE elevation, \% & 65.0 & 21.9 & 0.32 \\
H. pylori infection, \% & 30.6 & & - \\
Involved GI tract, \% (duplicates counted) & & 26.5 & - \\
Esophagus & 100 & 41.2 & - \\
Stomach & 0 & 76.5 & - \\
Small intestine & 0 & 17.4 & - \\
Colon & 0 & 0.9 & - \\
Ascites & 0 & & - \\
\hline
\end{tabular}

EoE, eosinophilic esophagitis; EGE, eosinophilic gastroenteritis; H. pylori, Helicobacter pylori; GI, gastrointestinal. * Allergy to seafoods was most common, followed by fruits in both groups.

Table 2. Laboratory test results for EoE and EGE

\begin{tabular}{lccl}
\hline & EoE $(n=181)$ & EGE $(n=34)$ & $p$ value \\
\hline Peripheral eosinophils, / $\mu \mathrm{L}$ & $259(289)$ & $234(667)$ & 0.92 \\
CRP, mg/dL & $0.05(0.09)$ & $0.04(0.09)$ & 0.37 \\
Total IgE, IU/mL & $137(376)$ & $246(1,383)$ & 0.09
\end{tabular}

Data are expressed as median (interquartile range). EoE, eosinophilic esophagitis; EGE, eosinophilic gastroenteritis.

was given to $20 \mathrm{EoE}$ patients who were nonresponsive to PPI therapy, and 12 showed clinical and histological remission. However, in those treated by topical steroid $(n=$ 23), all showed clinical and histological remission. None in the present cohort were treated by an elimination diet. Among the PPI-responsive EoE cases, $82.0 \%$ showed no symptomatic or histological relapse, while the remaining $18.0 \%$ experienced a relapse of esophageal eosinophilia even when PPI treatment was continued for $>1$ year. Of those with relapsing esophageal eosinophilia, 14 were treated with vonoprazan, and all of those available for assessment $(n=10)$ regained histological remission. Among 82 patients with no relapse of esophageal eosinophilia and who received maintenance PPI therapy, 32 discontinued the PPI treatment, of whom 21 later experienced relapse. However, in each case, esophageal eosinophilia was again controlled after readministration with the same dose of PPI. During the time course of the present study (20112019), no severe complications including esophageal stricture requiring esophageal dilatation treatment were encountered.

\section{Treatments for and Outcomes of Patients with EGE}

In the present cohort, oral glucocorticoid administration was mainly used in the EGE group (58.8\%) with initial doses ranging from 10 to $50 \mathrm{mg}$ daily, followed by a PPI (44.1\%) and antiallergic drugs (29.4\%), including montelukast $(n=8)$, epinastine $(n=2)$, and fexofenadine $(n=1)$ (duplicate counting) (Fig. 3 ).

Most patients responded to glucocorticoid therapy though some showed recurrence with a reduced dose. An 


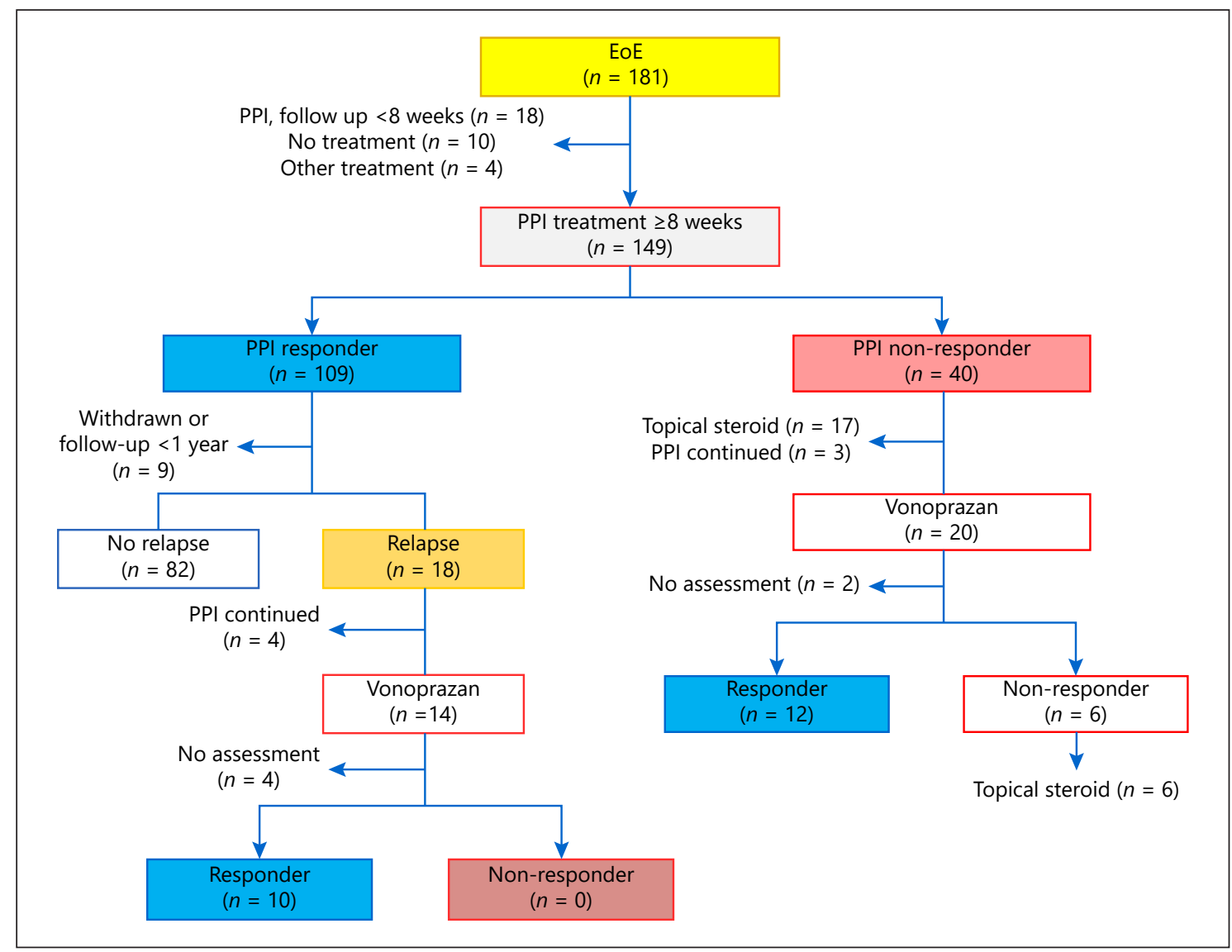

Fig. 2. Flow diagram showing enrollment of patients with EoE. EoE, eosinophilic esophagitis; PPI, proton pump inhibitor.

Fig. 3. Flow diagram showing enrollment of patients with EGE. EGE, eosinophilic gastroenteritis; PPI, proton pump inhibitor.

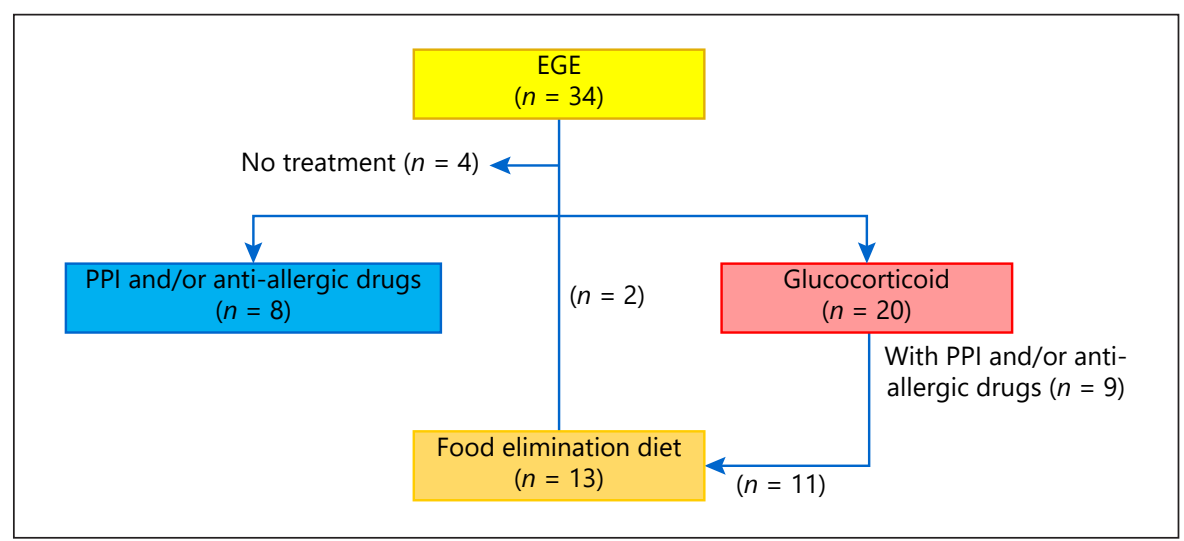

elimination diet is thought to be useful for EGE when the pathogenic antigenic food can be eliminated, the same as with EoE. Indeed, all 13 patients with EGE who were treated with a 6-food-elimination diet with avoidance of wheat, dairy products, eggs, nut/tree nuts, soy, and seafood showed improvement of GI symptoms. In addition, the responsible foods were successfully identified in 9 patients, and 7 were controlled in a state of remission without glucocorticoid therapy. Dairy products and soy were the most frequently identified allergenic foods. Notably, causal foods were not associated with the results of bloodspecific IgE antibodies in our cases (in submission). 


\section{Discussion}

EGID are a group of clinicopathological diseases with common pathological conditions, including exaggerated Th2 response to food or aeroallergens, which is considered to have an important role in development of EoE and EGE $[3,4]$. However, the relationship between the pathophysiology of EGID and distribution of eosinophilic infiltration in the GI tract remains unclear. In the present study, which included the largest number of EGID and EoE cases investigated in Japan, both similarities and differences regarding clinical characteristics in comparisons of EoE and EGE patients were noted.

There were remarkable differences between the EoE and EGE groups in regard to clinical features, including male/female ratio, BMI, and obesity ratio, though both groups were frequently accompanied with atopic conditions. Consistent with previous reports [20], the EoE group showed significant male predominance, while no gender difference was seen in the EGE group. In addition, patients with EoE had a higher obesity ratio as compared to those with EGE. These differences may be partially explained by gastroesophageal reflux associated with excess gastric acid secretion in young obese males as well as genetic factors [21]. A previous study of young adults without $H$. pylori infection showed that gastric acid secretion was higher in males than females [22]. In addition, obesity is a known risk factor for gastroesophageal reflux. Refluxed gastric acid may exacerbate the permeability of the esophageal epithelium, whereby food antigens easily penetrate that epithelium with decreased barrier function, causing esophageal eosinophilia. Furthermore, a recent study of adults in Japan showed that obesity and hiatal hernia may be related to esophageal eosinophilia [23], suggesting that those factors are involved in the pathogenesis of EoE as well as GERD. That speculation is supported by the good therapeutic effects of acid inhibitor administration in more than half of the present EoE cases.

Kinoshita et al. [9] reported clinical characteristics of patients with EoE $(n=26)$ and EGE $(n=144)$ in Japan diagnosed from 2004 to 2009 based on a questionnairebased nationwide survey conducted by the Japanese Society of Gastroenterology. At that time, EGE was more prevalent than EoE in Japan, as the latter was reported to be a very rare condition, with a calculated prevalence of $0.02 \%$ in endoscopy cases presented in 2011 [8]. However, more recent studies presented in 2019 have shown a prevalence of EoE of $0.34 \%$ in endoscopic-examined cases, suggesting that the prevalence in Japan is gradually increasing $[13,23]$ though the reason for that increase remains unknown. It is likely that the increasing prevalence of EoE is influenced by increased recognition of the condition, while a rapid decline in the prevalence of $H$. $p y$ lori infection in recent years in Japan has been speculated as another reason [24], as a recent systematic review and meta-analysis showed evidence for a significant association between $H$. pylori exposure and reduced odds of EoE development [25]. Consistently, a case control study previously conducted in Japan showed that the prevalence for $H$. pylori infection was significantly lower in EoE patients as compared with normal control subjects [26]. In contrast, no epidemiological studies performed in Japan have shown an increasing trend of EGE cases. In the present investigation, the number of enrolled cases of EGE during the study period was much fewer than EoE cases even though the rate of $H$. pylori infection was lower in the EGE patients as compared to those with EoE. The trend for increasing EoE cases may be affected by increased gastric acid secretion caused by the reduced $H$. pylori infection rate as well as increased prevalence of GERD in Japan, as discussed above.

Effective treatment strategies for EoE and EGE are substantially different. According to international consensus guidelines for EoE, current approaches consist of PPI administration, swallowed topical steroids, and elimination diet [17], with PPI treatment the most commonly employed first-line option in Japan. A recent systematic review showed that PPI use led to histological remission in $50.5 \%$ of patients with EoE [27], while over $70 \%$ of the present affected patients responded to initial PPI treatment, which may have been related to the mild clinical presentation generally seen in Japanese patients. Indeed, no cases with severe complication, such as esophageal stricture or perforation, were observed during the time course of our study. In addition, the majority who responded to PPI therapy maintained histological remission after at least 1 year of continuous administration, consistent with previously reported findings [28]. On the other hand, $>60 \%$ of our patients who discontinued PPI treatment had a relapse of symptoms and/or esophageal eosinophilia and needed retreatment with a PPI, suggesting that effective maintenance therapy and disease activity monitoring are necessary. Consistent with another previous study [29], vonoprazan was effective for not only cases of PPI nonresponsive EoE but also relapsing cases with continued PPI treatment. This drug has been shown to provide more potent acid-reducing effects as compared to conventional PPIs [30] and is a possible alternative for treatment of EoE. 
In contrast to EoE, there is a lack of randomized controlled trials to guide the choice of therapy for EGE [18]. As shown in the present study, glucocorticoid administration is the most widely used clinical treatment for EGE, and its effect is favorable for at least induction of remission [9]. However, the disease often relapses during tapering or after withdrawal of corticosteroid therapy, and there is also concern regarding adverse effects of longterm systemic corticosteroid use. Budesonide, a steroid with high first-pass metabolism, has been shown to be effective for the treatment of EGE using targeted combinations of opened, crushed, and intact enteric-coated capsules [31]. The better safety profile of budesonide compared to other glucocorticoids may be of particular benefit especially for steroid-dependent EGE cases.

While a food-elimination diet is a standard therapeutic option for patients with EoE, its effects for patients with EGE remain to be fully elucidated. Recently, we reported an adult EGE case successfully treated by an elimination diet [32]. In the current study, 13 patients with EGE were treated with a food-elimination diet, and responsible foods were successfully identified in 9 , of whom 7 remained controlled in a state of remission without glucocorticoid therapy. Those findings indicated that an elimination diet may be effective, especially for corticosteroid-dependent EGE cases. In contrast, allergy test-directed elimination diet which removes specific foods based on the results of allergy skin prick, blood-specific $\mathrm{IgE}$, and/or atopy patch testing shows quite low (32\%) in adult patients with EoE, suggesting that eosinophilic inflammation in the GI tract is likely independent of $\operatorname{IgE}$. Consistently, causal foods were not associated with the results of blood-specific IgE in our cases (in submission). Recently, biologic agents directed toward Th2 cytokines including IL-5, -4, and -13 have been used for the management of refractory allergic diseases such as asthma and atopic dermatitis and adopted in clinical trials to treat EoE and EGE. In addition, vedolizumab, a monoclonal antibody that selectively blocks $\alpha 4 \beta 7$ integrin, has been shown to be effective for $40 \%$ ( 2 of 5 patients) of refractory or steroid-dependent EGE. Nonetheless, larger-scale and appropriate clinical trials need be conducted to clarify the efficacy and safety profiles of elimination diet and molecular target therapy for EGE patients.

Limitations of this study include its retrospective design and patient enrollment from a single-tertiary referral center. Additionally, indication and selection criteria for treatment of patients with EGID were not uniformly determined and ultimately decided by the treating physician.

Eosinophilic Esophagitis and

Gastroenteritis
In conclusion, patients with EoE and EGE had different clinical characteristics and treatment strategies. Most with EoE responded well to acid-suppressive therapy with PPI or vonoprazan and then maintained histological remission after 1 year of PPI treatment. Although glucocorticoid administration was mainly used for EGE, a food-elimination diet showed beneficial effects for some of those cases.

\section{Statement of Ethics}

This study was approved by the ethics committee of the Shimane University Faculty of Medicine (No. 1930) and performed in accordance with the Declaration of Helsinki. All participants gave their written informed consent.

\section{Conflict of Interest Statement}

S.I. has received research funding from Takeda Pharmaceutical Co., Ltd., Astellas Pharma Inc., EA Pharma Co., Ltd., Janssen Pharmaceutical K.K., and ZERIA Pharmaceutical Co., Ltd., and lecture fees from Takeda Pharmaceutical Co., Ltd. The funding source had no role in the design, practice, or analysis of this study.

\section{Funding Sources}

This study was supported by a Grant-in-Aid for Scientific Research from the Ministry of Education, Culture, Sports, Science and Technology in Japan (18K07939).

\section{Author Contributions}

E.O. and N.I. designed the study, collected the data, and wrote the draft. S.I. made critical revisions. All authors reviewed and approved the final version of the article before submission.

References

1 Rothenberg ME. Eosinophilic gastrointestinal disorders (EGID). J Allergy Clin Immunol. 2004;113(1):11-29.

2 Kinoshita Y, Ishimura N, Oshima N, Mikami $\mathrm{H}$, Okimoto E, Jiao DJ, et al. Recent progress in the research of eosinophilic esophagitis and gastroenteritis. Digestion. 2016;93(1):7-12.

3 Caldwell JM, Collins MH, Stucke EM, Putnam PE, Franciosi JP, Kushner JP, et al. Histologic eosinophilic gastritis is a systemic disorder associated with blood and extragastric eosinophilia, TH2 immunity, and a unique gastric transcriptome. J Allergy Clin Immunol. 2014;134(5):1114-24. 
4 Shoda T, Morita H, Nomura I, Ishimura N, Ishihara S, Matsuda A, et al. Comparison of gene expression profiles in eosinophilic esophagitis (EoE) between Japan and Western countries. Allergol Int. 2015;64(3):260-5.

5 Khan S, Guo X, Liu T, Iqbal M, Jiang K, Zhu L, et al. An update on eosinophilic esophagitis: etiological factors, coexisting diseases, and complications. Digestion. 2020:1-15.

6 Mansoor E, Saleh MA, Cooper GS. Prevalence of eosinophilic gastroenteritis and colitis in a population-based study, from 2012 to 2017. Clin Gastroenterol Hepatol. 2017;15(11): 1733-41.

7 Navarro P, Arias Á, Arias-González L, Laserna-Mendieta EJ, Ruiz-Ponce M, Lucendo AJ. Systematic review with meta-analysis: the growing incidence and prevalence of eosinophilic oesophagitis in children and adults in population-based studies. Aliment Pharmacol Ther. 2019;49(9):1116-25.

8 Fujishiro H, Amano Y, Kushiyama Y, Ishihara S, Kinoshita Y. Eosinophilic esophagitis investigated by upper gastrointestinal endoscopy in Japanese patients. J Gastroenterol. 2011; 46(9):1142-4.

9 Kinoshita Y, Furuta K, Ishimaura N, Ishihara S, Sato S, Maruyama R, et al. Clinical characteristics of Japanese patients with eosinophilic esophagitis and eosinophilic gastroenteritis. J Gastroenterol. 2013;48(3):333-9.

10 Ito J, Fujiwara T, Kojima R, Nomura I. Racial differences in eosinophilic gastrointestinal disorders among Caucasian and Asian. Allergol Int. 2015;64(3):253-9.

11 Adachi K, Mishiro T, Tanaka S, Kinoshita Y. Suitable biopsy site for detection of esophageal eosinophilia in eosinophilic esophagitis suspected cases. Dig Endosc. 2016;28(2):13944.

12 Kinoshita Y, Ishimura N, Mishiro T, Ishihara S, Adachi K. Diagnosis and treatment of eosinophilic esophagitis in Japan. Esophagus. 2016;14(1):66-75.

13 Ishimura N, Kinoshita Y. Eosinophilic esophagitis in Japan: focus on response to acid suppressive therapy. J Gastroenterol Hepatol. 2018;33(5):1016-22.

14 Ishimura N, Shimura S, Jiao D, Mikami H, Okimoto E, Uno G, et al. Clinical features of eosinophilic esophagitis: differences between Asian and Western populations. J Gastroenterol Hepatol. 2015;30(Suppl 1):71-7.
15 Jiao D, Ishimura N, Maruyama R, Ishikawa N, Nagase M, Oshima N, et al. Similarities and differences among eosinophilic esophagitis, proton-pump inhibitor-responsive esophageal eosinophilia, and reflux esophagitis: comparisons of clinical, endoscopic, and histopathological findings in Japanese patients. J Gastroenterol. 2017;52(2):203-10.

16 Toya Y, Kumei T, Yamada S, Akasaka R, Yanai S, Nakamura S, et al. Eosinophilic esophagitis with a severe stenosis: report of a Japanese case. Clin J Gastroenterol. 2020;13(5): 708-712. http://dx.doi.org/10.1007/s12328020-01145-0.

17 Lucendo AJ, Molina-Infante J, Arias Á, von Arnim U, Bredenoord AJ, Bussmann C, et al Guidelines on eosinophilic esophagitis: evidence-based statements and recommendations for diagnosis and management in children and adults. United European Gastroenterol J. 2017;5(3):335-58.

18 Ishihara S, Kinoshita Y, Schoepfer A. Eosinophilic esophagitis, eosinophilic gastroenteritis, and eosinophilic colitis: common mechanisms and differences between East and West. Inflamm Intest Dis. 2016;1(2):63-9.

19 Diagnostic criteria for eosinophilic gastrointestinal disorders: Japan Intractable Diseases Information Center, https://www.nanbyou. or.jp/ entry/3935 (in Japanese).

20 Kinoshita Y, Ishimura N, Oshima N, Ishihara S. Systematic review: eosinophilic esophagitis in Asian countries. World J Gastroenterol. 2015;21(27):8433-40.

21 Kottyan LC, Rothenberg ME. Genetics of eosinophilic esophagitis. Mucosal Immunol. 2017;10(3):580-8.

22 Ishimura N, Owada Y, Aimi M, Oshima T, Kamada $\mathrm{T}$, Inoue $\mathrm{K}$, et al. No increase in gastric acid secretion in healthy Japanese over the past two decades. J Gastroenterol. 2015;50(8): $844-52$.

23 Tanaka F, Fukumoto S, Morisaki T, Otani K, Hosomi S, Nagami Y, et al. Obesity and hiatal hernia may be non-allergic risk factors for esophageal eosinophilia in Japanese adults. Esophagus. 2019;16(3):309-15.

24 Inoue M. Changing epidemiology of Helicobacter pylori in Japan. Gastric Cancer. 2017; 20(Suppl 1):3-7.
25 Shah SC, Tepler A, Peek RM Jr, Colombel JF, Hirano I, Narula N. Association between Helicobacter pylori exposure and decreased odds of eosinophilic esophagitis-a systematic review and meta-analysis. Clin Gastroenterol Hepatol. 2019;17(11):2185-e3.

26 Furuta K, Adachi K, Aimi M, Ishimura N, Sato S, Ishihara S, et al. Case-control study of association of eosinophilic gastrointestinal disorders with Helicobacter pylori infection in Japan. J Clin Biochem Nutr. 2013;53(1): 60-2.

27 Lucendo AJ, Arias Á, Molina-Infante J. Efficacy of proton pump inhibitor drugs for inducing clinical and histologic remission in patients with symptomatic esophageal eosinophilia: a systematic review and meta-analysis. Clin Gastroenterol Hepatol. 2016;14(1):1322.e1.

28 Molina-Infante J, Rodriguez-Sanchez J, Martinek J, van Rhijn BD, Krajciova J, Rivas $\mathrm{MD}$, et al. Long-term loss of response in proton pump inhibitor-responsive esophageal eosinophilia is uncommon and influenced by CYP2C19 genotype and rhinoconjunctivitis. Am J Gastroenterol. 2015; 110(11):1567-75.

29 Ishimura N, Ishihara S, Kinoshita Y. Sustained acid suppression by potassium-competitive acid blocker (P-CAB) may be an attractive treatment candidate for patients with eosinophilic esophagitis. Am J Gastroenterol. 2016;111(8):1203-4.

30 Kinoshita Y, Ishimura N, Ishihara S. Management of GERD: are potassium-competitive acid blockers superior to proton pump inhibitors? Am J Gastroenterol. 2018;113(10): 1417-9.

31 Kennedy K, Muir AB, Grossman A, BrownWhitehorn T, Cianferoni A, Spergel JM, et al. Modified oral enteric-coated budesonide regimens to treat pediatric eosinophilic gastroenteritis, a single center experience. J Allergy Clin Immunol Pract. 2019 Jul-Aug;7(6): 2059-61.

32 Okimoto E, Ishimura N, Okada M, Mikami $\mathrm{H}$, Sonoyama H, Ishikawa N, et al. Successful food-elimination diet in an adult with eosinophilic gastroenteritis. ACG Case Rep J. 2018; 5:e38. 